\title{
Research Paper \\ Quantitative and Qualitative Monitoring of Airborne Bacteria and Fungi and Their Relationship with Environmental Parameters in Two Selected Primary Schools
}

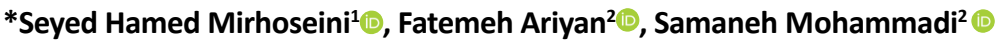 \\ 1. Department of Environmental Health, School of Health, Arak University of Medical Sciences, Arak, Iran. \\ 2. Student Research Committee, School of Health, Arak University of Medical Sciences, Arak, Iran.
}

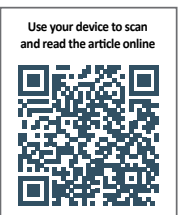

Crtation: Mirhoseini SH, Ariyan F, Mohammadi S. [Quantitative and Qualitative Monitoring of Airborne Bacteria and Fungi and Their Relationship with Environmental Parameters in Two Selected Primary Schools (Persian)]. Journal of Arak University of Medical Sciences (JAMS). 2020; 22(6):242-251. https://doi.org/10.32598/JAMS.22.6.5931.1

dois https://doi.org/10.32598/JAMS.22.6.5931.1

Key words: Bioaerosol, Primary school, Particulate Matter, Bacteria, Fungi

\section{AB STRACT}

Background and Aim The assessment of indoor air quality and detection of its microbial pollutants in classrooms is very important because of the presence of children sensitive to these pollutants. The aim of this study was to determine the concentration and characterization of dominant species of biological aerosols and their relationship with environmental factors in two selected primary schools in Arak, Iran. Methods \& Materials This cross-sectional study was conducted at two primary schools in Arak, Iran in Fall 2018. Indoor air sampling was performed using single-stage Andersen microbial sampler (at flow rate of 28.3 liters/min) containing bacterial and fungal culture media. The effects of suspended Particulate Matter (PM) and environmental parameters (temperature and humidity) on the density of bioaerosols were evaluated.

Ethical Considerations This study with an ethics code of IR.ARAKMU.REC.1397.76 was approved by the Research Ethics Committee at Arak University of Medical Sciences.

Results The overall mean density of indoor bacteria and fungi was $448 \mathrm{cfu} / \mathrm{m}^{3}$ and $394 \mathrm{cfu} / \mathrm{m}^{3}$, while the mean density of outdoor bacteria and fungi was $210 \mathrm{cfu} / \mathrm{m}^{3}$ and $127 \mathrm{cfu} / \mathrm{m}^{3}$, respectively. There was a positive correlation between indoor density of airborne bacteria and suspected PM concentrations (PM 10 and PM 2.5), and between PM2.5 concentration and indoor fungal density ( $P<0.05)$. Penicillium (40\%), Cladosporium (19\%) and Aspergillus (16\%) were dominant species of fungi, while Staphylococcus (42\%), Micrococcus (28\%), Bacillus (21\%) were the dominant species of bacteria.

Conclusion The age and type of building and the density of students in a classroom are the main factors in increasing the concentration of bioaerosols.

\section{Extended Abstract}

\section{Introduction}

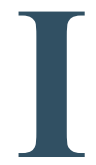

$\mathrm{n}$ recent years, exposure to bioaerosols has been of great importance due to its associated health problems. The World Health Organization has warned of the health implications of poor indoor air quality and incidence of infectious, respiratory and allergic diseases. The classroom is an environment where students spend most of their day. Inside the classroom, students are exposed to airborne physical, chemical, and microbial agents. The assessment of indoor air quality and detection of microbial pollutants in classrooms is very important because of the presence of children sensitive to these pollutants. The aim of this study was to determine the

\section{* Corresponding Author:}

Seyed Hamed Mirhoseini, PhD.

Address: Department of Environmental Health, School of Health, Arak University of Medical Sciences, Arak, Iran.

Tel: +98 (918) 3495575

E-mail: dr.mirhoseini@arakmu.ac.ir and hmirhossaini@gmail.com 
concentration and characterization of dominant species of biological aerosols and their relationship with environmental factors in two selected primary schools in Arak, Iran.

\section{Methods and Materials}

This cross-sectional study was conducted at two primary schools in Arak, Iran in Fall 2018. Indoor air sampling was performed using single-stage Andersen microbial sampler (at flow rate of 28.3 liters.min) containing bacterial and fungal culture media. Indoor air sampling was carried out during regular class activity hours at a height of $1.5 \mathrm{~m}$ above ground level. At the same time, the effects of suspended Particulate Matter (PM) and environmental parameters (temperature and humidity) on the density of bioaerosols were evaluated. In order to determine the ratio of indoor to outdoor concentrations, sampling of school yard air was performed simultaneously as an outdoor sample. Bacterial media were incubated at $37^{\circ} \mathrm{C}$ for $24-48 \mathrm{~h}$, and plates of fungal samples were kept at ambient air for 5-7 days. The number of colonies was then counted and reported per CFU.m ${ }^{3}$. Bacterial colonies were identified by biochemical tests and fungal colonies were identified through macroscopic morphological features and microscopic observations. Kruskal-Wallis test was used to compare the concentration of bioaerosols in different environments, and Spearman's rank correlation test was used to investigate the relationship between different parameters.

\section{Results}

The overall mean density of indoor bacteria and fungi was 448 cfu.m3 and 394 cfu.m ${ }^{3}$, while the mean density of outdoor bacteria and fungi was $210 \mathrm{cfu} \cdot \mathrm{m}^{3}$ and $127 \mathrm{cfu} . \mathrm{m}^{3}$, respectively. The highest and the lowest mean bacterial density belonged to the second floor classroom of School No. 2 $\left(559 \pm 141\right.$ cfu. $\left.^{3}\right)$ and the first floor classroom of School No. $1\left(293 \pm 170\right.$ cfu. $\left.\mathrm{m}^{3}\right)$, respectively. Moreover, the first floor classroom of School No. $1\left(63 \pm 25\right.$ cfu.m $\left.{ }^{3}\right)$ and the first floor classroom of School No. 2 (132 \pm 98 cfu.m ${ }^{3}$ ) had the lowest and highest mean fungal density, respectively, in the air of different areas (Figure 1).

Statistical analysis showed that there was a significant difference between the indoor concentrations of airborne bacteria and fungi $(\mathrm{P}<0.05)$, but not between their outdoor concentrations $(\mathrm{P}>0.05)$. Mann-Whitney $\mathrm{U}$ test results showed that the mean concentrations of PM10 and $\mathrm{PM}_{2.5}$ in outdoor air were significantly higher than in indoor air (Table 1 ). There was a positive correlation between indoor density of airborne bacteria and suspected PM concentrations $\left(\mathrm{PM}_{10}\right.$ and $\left.\mathrm{PM}_{2.5}\right)$, and between $\mathrm{PM}_{2.5}$ concentration and indoor fungal density $(\mathrm{P}<0.05)$. Humidity and density of indoor airborne bacteria and fungi were directly correlated with each other, but no relationship between temperature and density of biological aerosols was reported (Table 2). Penicillium (40\%), Cladosporium (19\%) and Aspergillus (16\%) were dominant species of fungi, while Staphylococcus (42\%), Micrococcus (28\%), Bacillus (21\%) were the dominant species of bacteria.

\section{Discussion}

A review of past studies shows that a wide range of bioaerosol concentrations have been reported in the classrooms. Some of the studies are consistent with our study $[12,13]$. A number of studies have reported higher levels of bioaerosol density. For example, in studies conducted in two girls' high schools of Eslamhshar county in Tehran [2], in 73 classrooms of 20 elementary schools located in Porto, Portugal in winter [10], and in primary schools in Gondar, Ethiopia [14], higher bioaerosol density were reported. Various factors such as: sampling season, environmental and climatic conditions, internal sources of bioaerosol production and differences in study design (sample size, sequence and duration of sampling) are the main reasons for the differences in

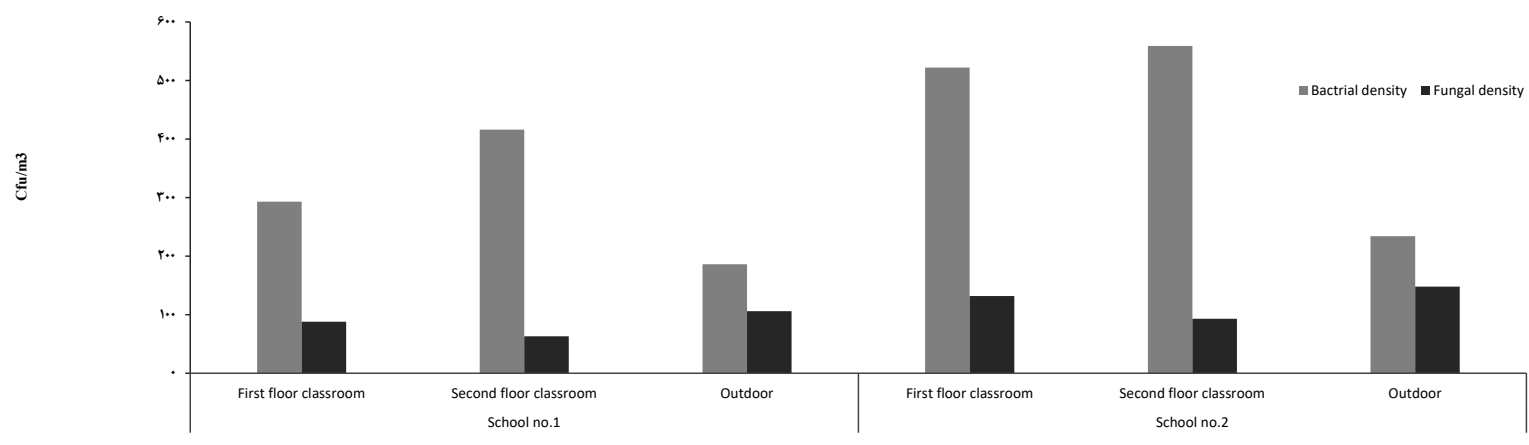

Figure 1. Comparing the mean density of bacteria and fungi (cfu/m3) in different air sampling sites 
Table 1. Mean concentrations of PM10 and PM2.5 $(\mu \mathrm{g} / \mathrm{m} 3)$ and mean temperature and humidity of sampling sites

\begin{tabular}{cccccc}
\hline School & Sampling site & Temperature (C'1) & Humidity (\%) & PM10 & PM2.5 \\
\hline \multirow{3}{*}{ School No. 1} & first floor classroom & $20 / 0 \pm 1 / 8$ & $2 / 34 \pm 0 / 2$ & $47 \pm 21$ & $28 \pm 14$ \\
& second floor classroom & $21 / 0 \pm 6 / 7$ & $35 / 1 \pm 6 / 2$ & $36 \pm 11$ & $19 \pm 8$ \\
& outdoor & $18 / 4 \pm 0 / 6$ & $4 / 36 \pm 1 / 8$ & $84 \pm 22$ & $47 \pm 10$ \\
School No. 2 & first floor classroom & $22 / 2 \pm 0 / 9$ & $39 / 3 \pm 4 / 2$ & $68 \pm 26$ & $38 \pm 18$ \\
& second floor classroom & $21 / 6 \pm 0 / 5$ & $40 / 2 \pm 9 / 9$ & $75 \pm 31$ & $44 \pm 29$ \\
\hline
\end{tabular}

Table 2. Spearman rank correlation matrix for different study parameters

\begin{tabular}{|c|c|c|c|c|c|c|}
\hline & Density of Bacteria & Density of Fungi & PM10 & PM2.5 & Temperature & Humidity \\
\hline Density of bacteria & 1 & & & & & \\
\hline Density of fungi & $-0 / 008$ & 1 & & & & \\
\hline PM10 & *0/282 & $* 0 / 462$ & 1 & & & \\
\hline PM2.5 & $* 0 / 324$ & $* 0 / 301$ & *0/92 & 1 & & \\
\hline Temperature & $0 / 123$ & $0 / 213$ & 0/003 & $-0 / 048$ & 1 & \\
\hline Humidity & $* 0 / 298$ & $* 0 / 363$ & $0 / 002$ & $-0 / 063$ & $-0 / 016$ & 1 \\
\hline
\end{tabular}

*Significant at $\mathrm{P}<0.05$.

the results between our study and other studies. In this study, the I.O ratios varied from 1.4 to 5.6 for bacterial aerosols and from 0.4 to 1.2 for fungal aerosols. The highest ratio for bacteria was found in School n. 2(2.4), indicating that the origin of airborne bacteria in the classroom was internal. The dominant bacterial and fungal species identified in this study are consistent with results obtained from indoor air isolates of school in other studies [4, 22, 23]. Staphylococcus as a natural flora of the skin and nose can cause a wide range of diseases and infections, especially in children. Corynebacterium is also known to be a contributing factor to nosocomial infections, especially in children [24]. The dominant fungal species reported in this study are the most important allergens that have detrimental effects on human health, especially children and students in the classroom [19].

\section{Ethical Considerations}

\section{Compliance with ethical guidelines}

This study with an ethics code of IR.ARAKMU. REC.1397.76 was approved by the Research Ethics Committee at Arak University of Medical Sciences.

\section{Funding}

This study was extracted from a research proposal approved by Arak University of Medical Sciences (code:
3079). The authors would like to thank the Vice-Chancellor for Research and Student Research Committee of Arak University of Medical Sciences for their valuable spiritual and financial support.

\section{Authors' contributions}

All authors met the writing standards based on the recommendations of the International Committee of Medical Journal Editors (ICMJE), and they had equal attribution in preparing the paper.

\section{Conflicts of interest}

The authors declare no conflict of interest. 


\title{
يايش كمّى و كيفى باكترىها و قارجهاى منتقله توسط هوا و ارتباط آنها با عوامل محيطى در دو مدرسه ابتدايى منتخب شيفر ارئري
}

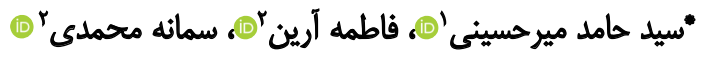 \\ ا. كروه مهندسى بهداشت محيط، دانشكده بهداشت دانشكاه علوم يز شكى اراك، اراكى ايران.

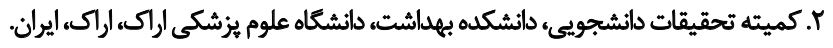

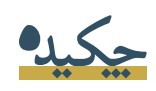

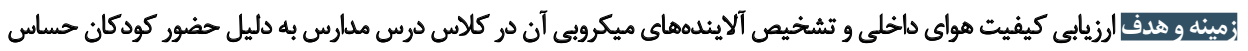

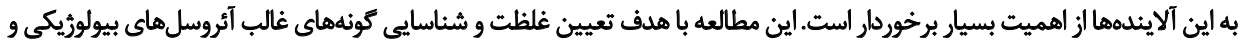

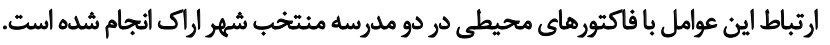

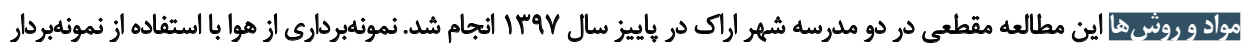

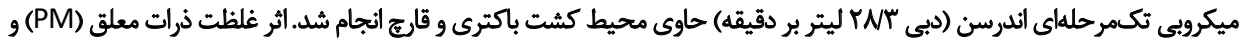

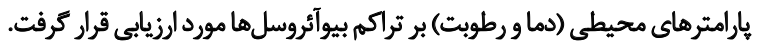

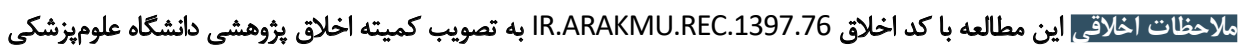
اراك رسيده است.

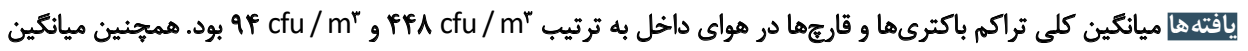

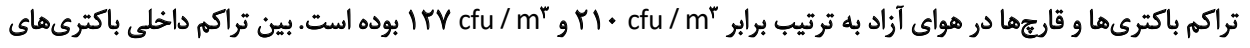

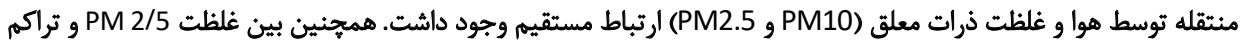

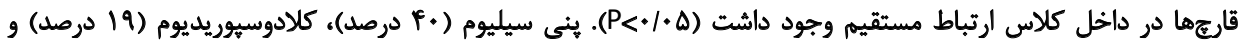

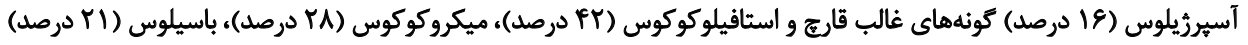

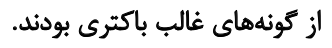

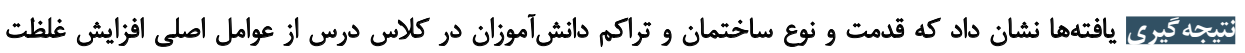

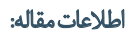

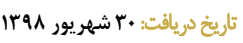

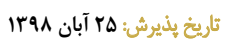
تاريخ ايتشار: كا بهمن

كلاس درس مدارس محيطى است كه دانشآموزان بعد از

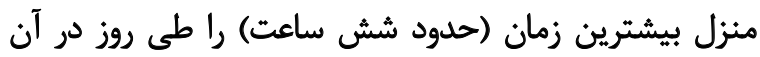

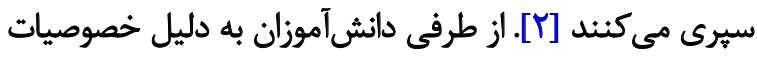

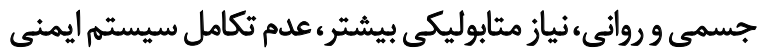

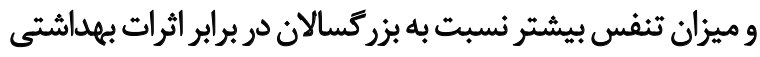

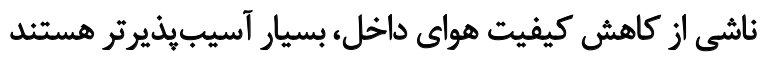

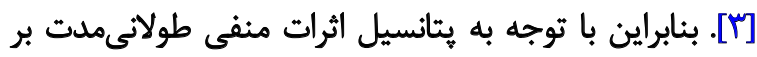

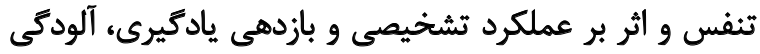

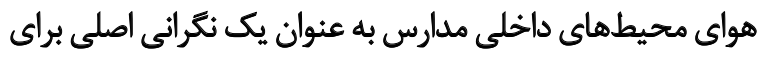

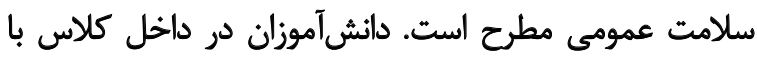

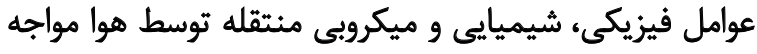

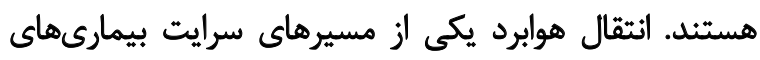

\begin{abstract}
قراركرفتن در معرض ذرات موجود در هوا (PM) يكى از

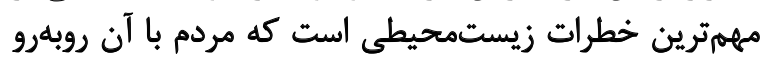

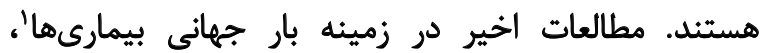

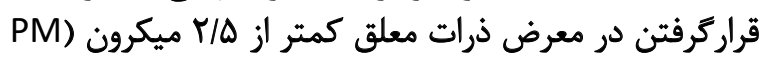

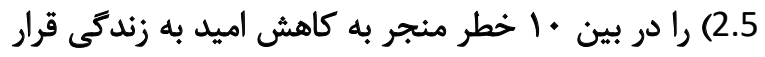

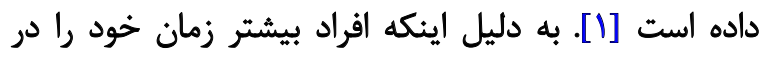

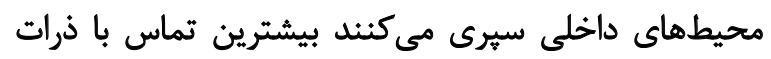

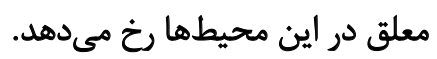

1. Global bureden of disease

*ويسئده مسينول: سيد حامد مير حسينى نئين

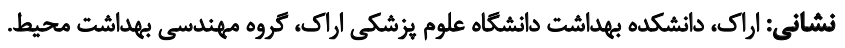
تلفي: لِيت الكترونيكي: dr.mirhoseini@arakmu.ac.ir; hmirhossaini@gmail.com 
مواد و روشىها

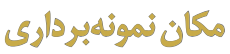

اين يُوهش توصيفى مقطعى در دو مدرسه ابتدايى نوساز

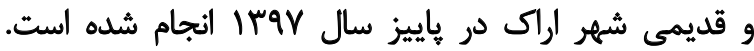

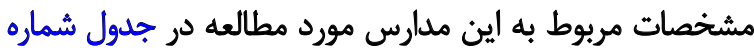

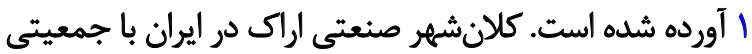

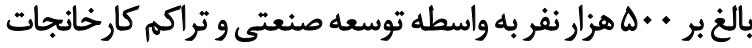

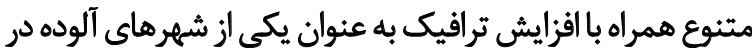

$$
\text { كشور شناخته مىشود. }
$$

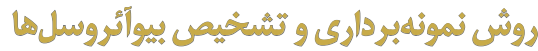

نمونهبردارى فعال از هواى داخل دو كلاس منتخب در هر

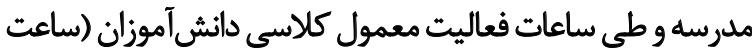
اخلجام شد. (IT-1.

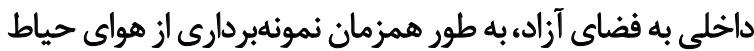

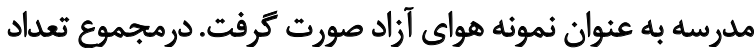

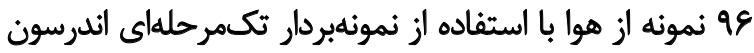

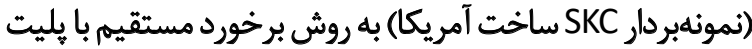

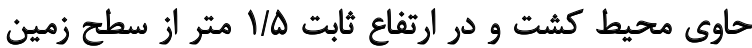

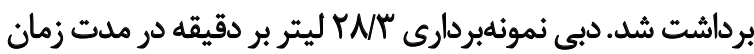

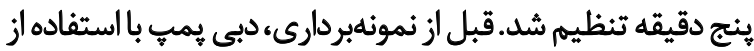

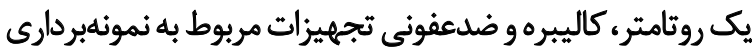

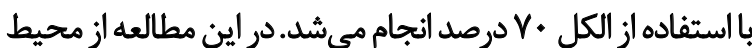

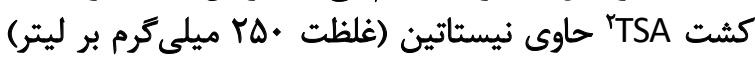

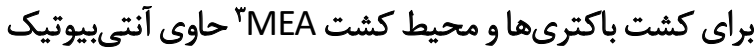

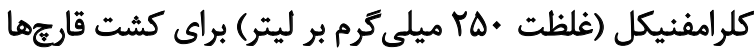

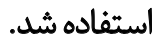

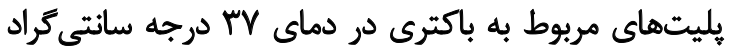

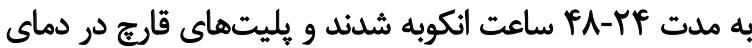

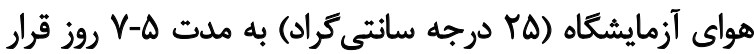

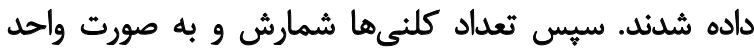

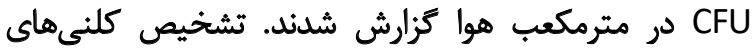

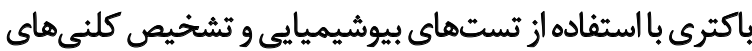

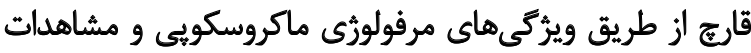
ميكروسكويى صورت كرفت [11]

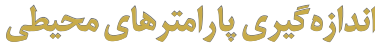

در طول مدت نمونهبردارى از هوا، همز مان بارامترهاى محيطى موري

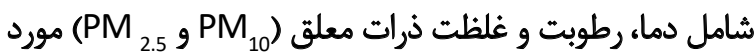

2. Tryptic Soy Agar

3. Malt Extract Agar
عفونى در دانشآموز محسوب مي شود. نوع و تراكم بيوأئروسلها

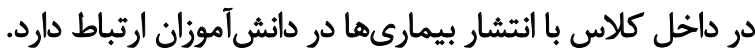

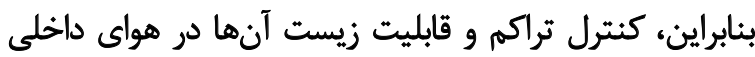

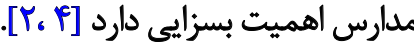

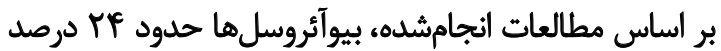

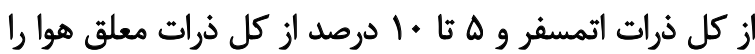

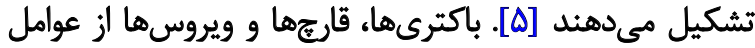

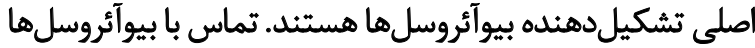

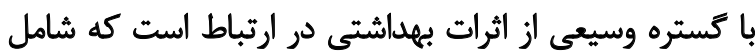

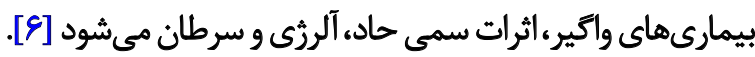

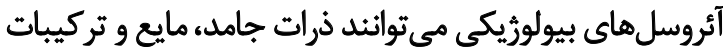

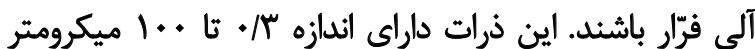

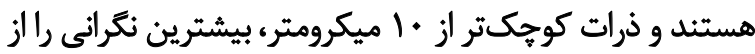

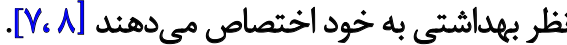
افزايش عايقبندى ساختمان همراه با تهويه ضعيف، محيطهايى را با تماس بالا با بيوآئروسلها ائمان ايجاد كرده است.

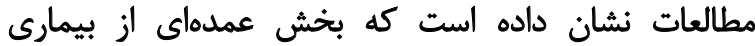

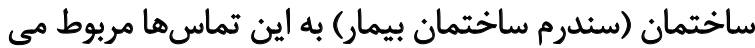

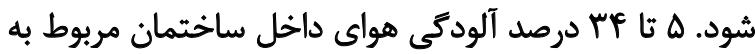

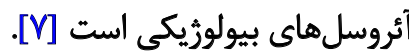
استافيلوكى اورئوس مقاوم به متىسيلين، اسينتوباكتر،

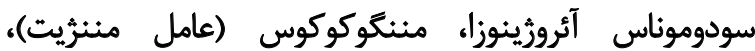

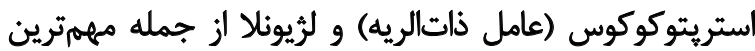

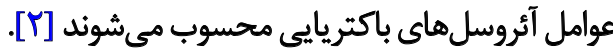
مايكوتوكسينها واندوتوكسينهاي توليدشده به وسيله قارجها

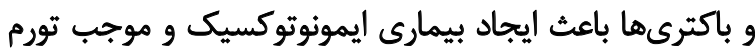

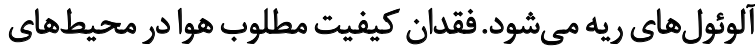

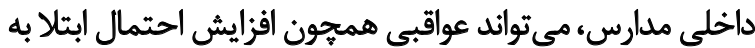

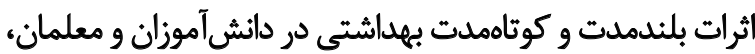

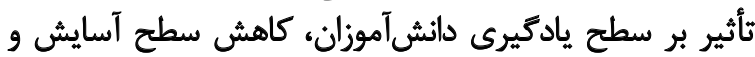

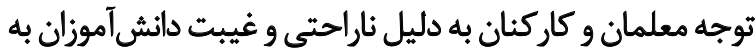

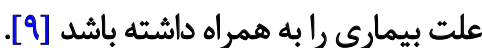

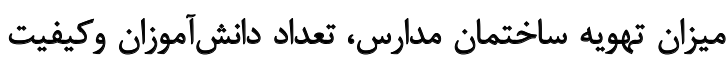
هواى اطراف مدارس برخى از اصلى ترين عوامل تأثير كذار بران بركيفيت

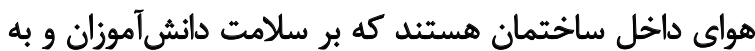

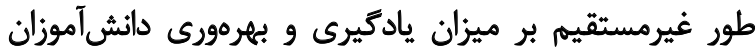

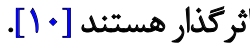

بنابراين اين مطالعه با هدف تعيين تراكم و نوع آئروسلهاى

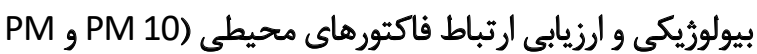

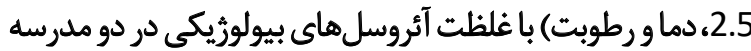
منتخب شهر اراك انجام شده است. 


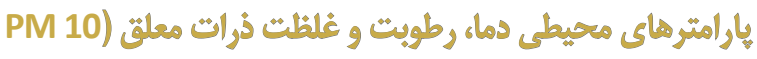
(PM 2/5

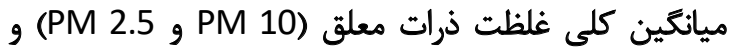

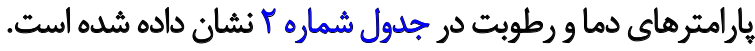

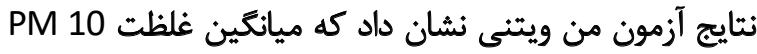

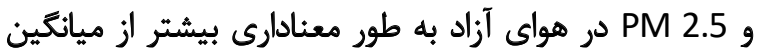

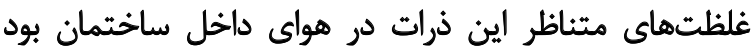

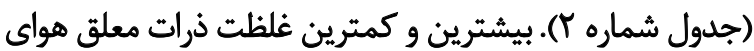

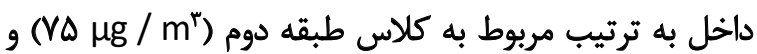

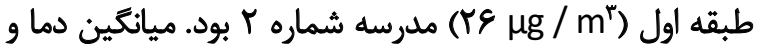

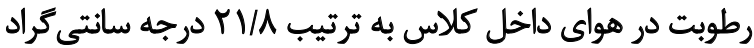

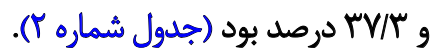

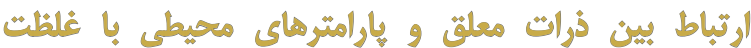

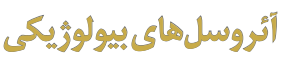

جدول شماره ب ضريب همبستخى رتبهاي اسبيرمن را بين

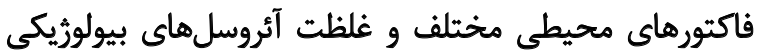

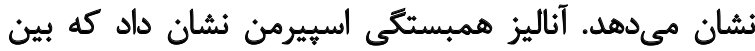

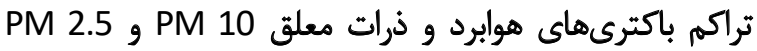

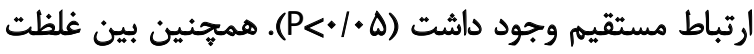

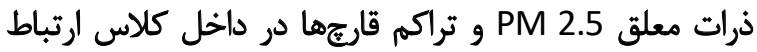

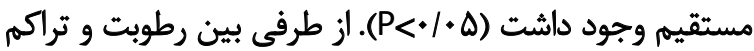

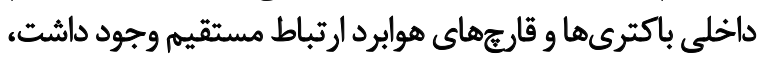

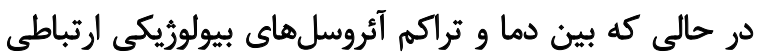
مشاهده نشد (جدول شماره بَ).

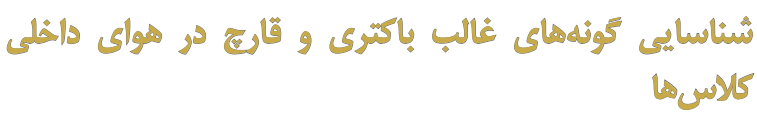

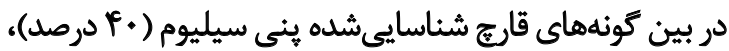

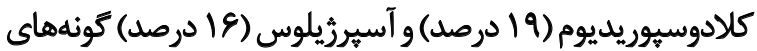

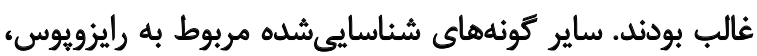

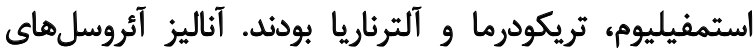

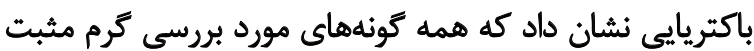

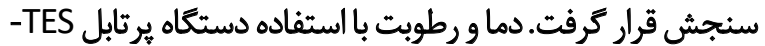

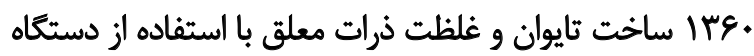
Dust track TSI Model 8520 ساخت آمريكا تعيين شاندان قبل از نمونهبردارى، دستكاه توسط كاليبراتور زيرو فيلتر مختص

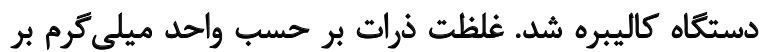

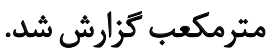

\section{Lasls}

آناليز نتايج با استفاده از نسخه + ب نرمافزار SPSS نسخه شماره

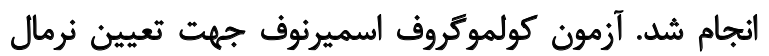

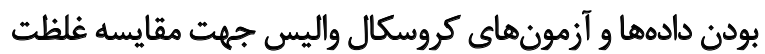
بيوآئروسل ها در محيطهاي مختلف استفاده شدئ هميجنين آناليز

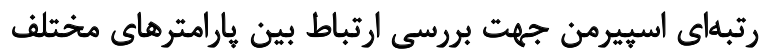

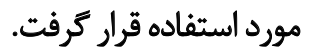

يافتهها

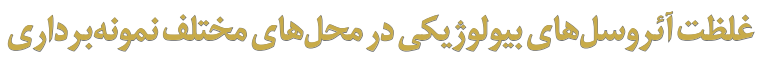

در اين مطالعه غلظتهاى داخلى باكترىهاي هوابرد در

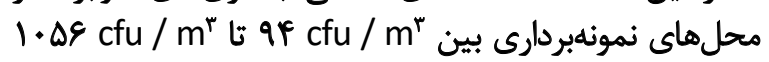

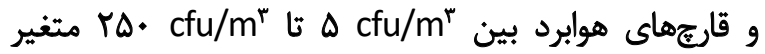

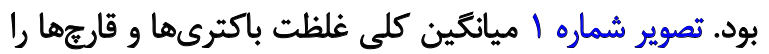

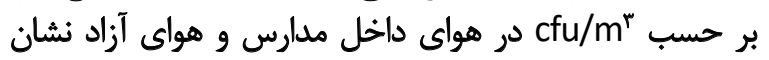

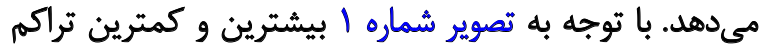

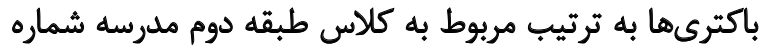

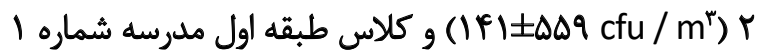

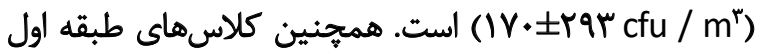

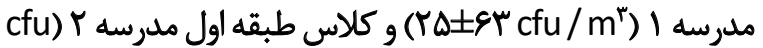

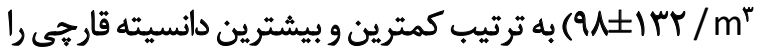
در هواى محل هاى مختلف نمونهبردارى داشتيند.

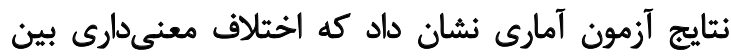

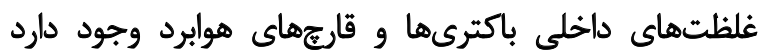

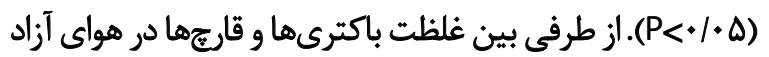

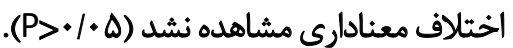

جدول ا. مشخصات مربوط مدارس موردمطالعه

\begin{tabular}{|c|c|c|c|c|c|}
\hline نوع سيستم تهويه & مساحت (m) & تعداد دانش أموزان & محل نمونهبردارى & قدمث ساخت & نام مدرسه \\
\hline هواساز مركزى & $\Delta$. & $m$ & كالاس طبقه اول & \multirow{2}{*}{ r } & \multirow{2}{*}{ مدرسه| } \\
\hline هواساز مركزى & $\Delta$ & ru & كلاس طبقه دوم & & \\
\hline تهويه طبيعى & m & f. & كالاس طبقه اول & \multirow{2}{*}{10} & \multirow{2}{*}{ ملرud r } \\
\hline تهويه طبيعى & $r$ & et & كلاس طبقه دوم & & \\
\hline
\end{tabular}




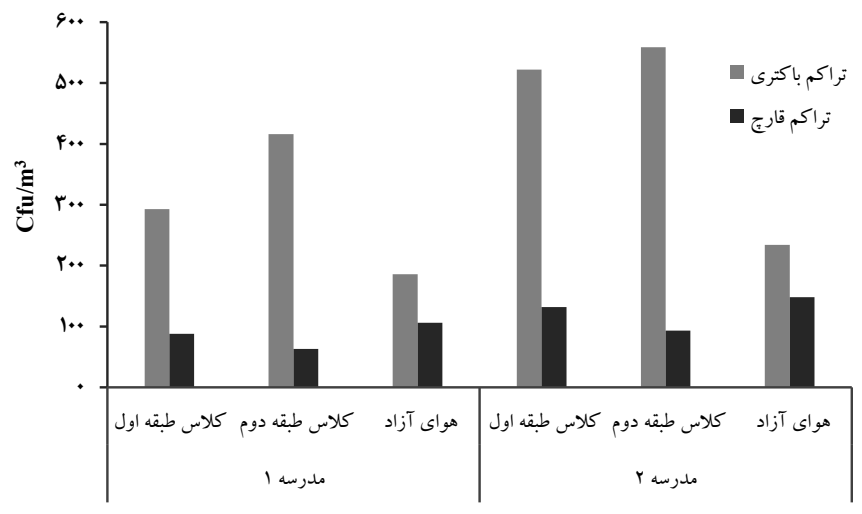

تصوير ا. مقايسه ميانكين تراكم باكترىها و قارعها (cfu/m) در هواي محل هاى مختلف نمونهبردارى

برخوردار است. نتايج اين يُروهش نشان داد كه ميانكين كلى داري

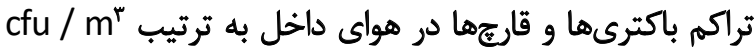

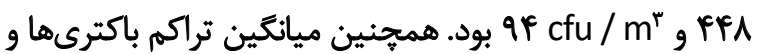

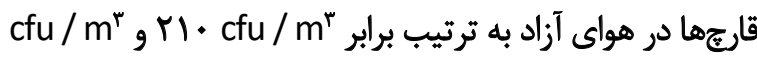

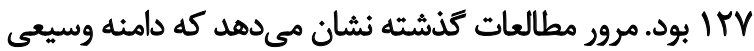

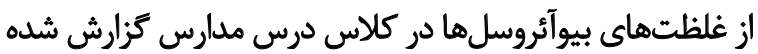

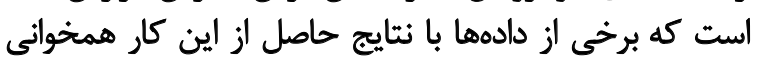

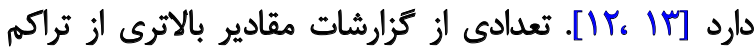

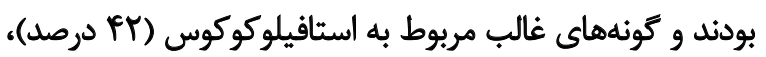

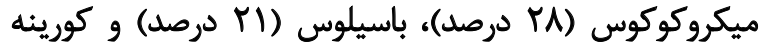

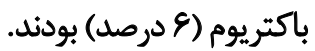
بحث

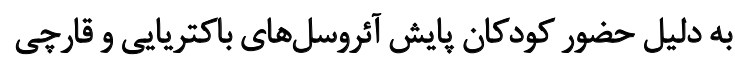

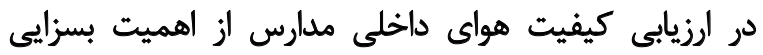

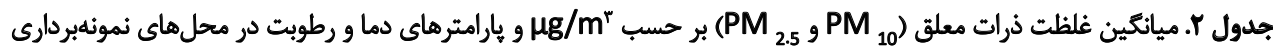

\begin{tabular}{|c|c|c|c|c|c|}
\hline $\mathbf{P M}_{2.5}$ & $\mathbf{P M}_{10}$ & رطوبت (\%) & Sما & محل نمونهبردارى & ن ام مدرسه \\
\hline rutif & $r v \pm r$ & $r / \Gamma \varphi / \pm \cdot / r$ & $r+/ \pm \pm / A$ & كلاس طبقه اول & \\
\hline $19 \pm 1$ & $r q \pm 11$ & $r \Delta / \perp \pm g / r$ & $r V / \pm E / V$ & كلاس طبقه دوم & مدرسه 1 \\
\hline$r v \pm 1$. & $A F \pm r r$ & $r / r q \pm 1 / A$ & $W F \cdot \pm / q$ & هواى آزاد & \\
\hline$r \Lambda \pm ו \Lambda$ & TSYAt & $r q / r \pm F / r$ & $r r / r \cdot \pm / q$ & كلاس طبفه اول & \\
\hline pqtrq & 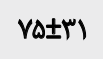 & $r \cdot / r \pm q q$ & $r V / q \cdot \pm / \Delta$ & كلاس طبقه دوم & مدرسه Y \\
\hline 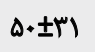 & raw & $M / \cdot \pm N A$ & $19 / r \cdot \pm / f$ & هواى آزاد & \\
\hline
\end{tabular}

جدول ؟. مائريس ضريب همبستىى رثبهاي اسييرمن بين بارامترهاى مخثلف

\begin{tabular}{|c|c|c|c|c|c|c|}
\hline رطوبت & دما & $\mathrm{PM}_{2.5}$ & $P M_{10}$ & تراكم قارع & تراكم باكترى & \\
\hline & & & & & 1 & تراكم باكترى \\
\hline & & & & 1 & $-* 1+* 1$ & تراكم قارج \\
\hline & & & 1 & . &.$/ r A r^{*}$ & PM 10 \\
\hline & & 1 & ./9r" & $\cdot r+1^{*}$ & . MTrP" & PM 2.5 \\
\hline & 1 & $-.1 \cdot+\Lambda$ &.$/ . r r$ & . MIr &.$/ 1 \pi$ & Las \\
\hline 1 & -.1 .18 &.- .1 .94 &.$/ . r$ & T &.$/ u^{*}$ & رطوبت \\
\hline
\end{tabular}


بين ميانكين غلظت قارجها در محيط داخل و هواى آزاد تفاوت

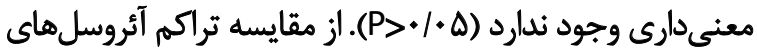

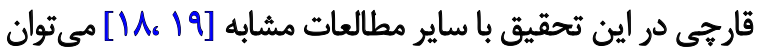

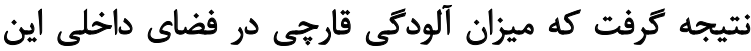
مدارس در طى دوره نمونهبردارى بالا نيست. با توجه به نتايج ارائهشده در جدول شماره r ب، ميانكين غلظت

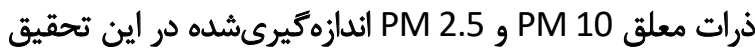

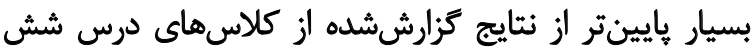

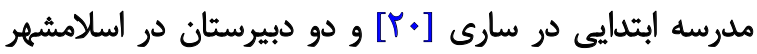

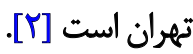

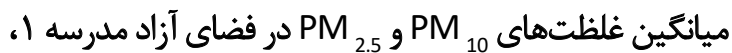

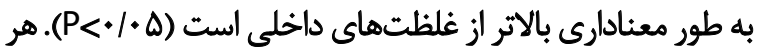

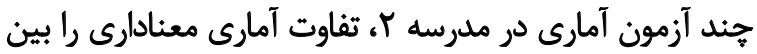

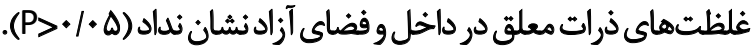
مدارس مورد بررسى در اين تحقيق در مجاتئي

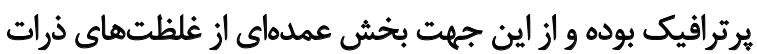
معلق در فضاى داخلى ناشى از تردد خودروهاست.

آناليز همبستكى اسييرمن نشان داد كه بين تراكم بيوآئروسلها

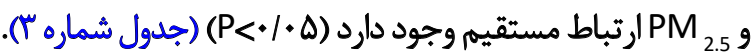

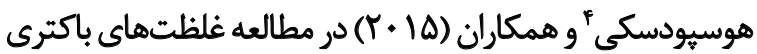

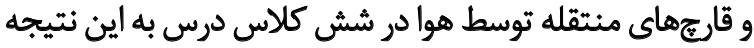

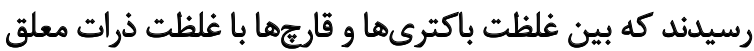

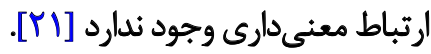

آناليز همبستگى اسيرمن ارتباط ضعيفى را بين غلظت

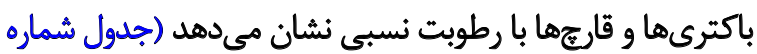

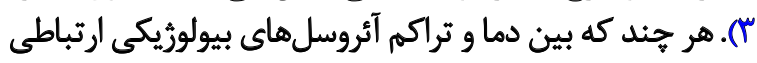

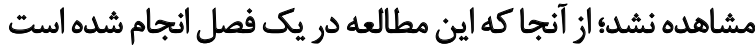

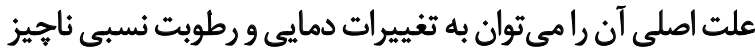

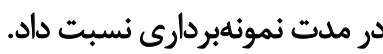

كونههاى غالب باكتريايى و قارج شناسايىشده در اين يرؤهش

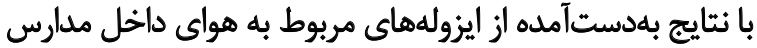

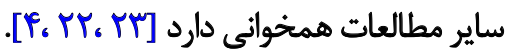

باكترى استافيلوكوكوس به عنوان فلور طبيعى يوست و بينى

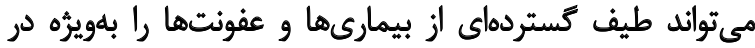

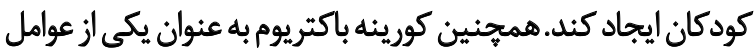

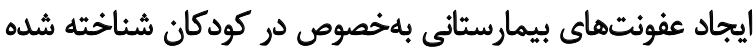

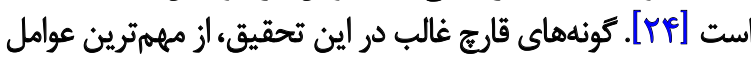

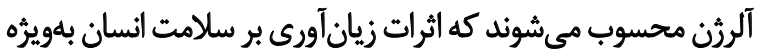

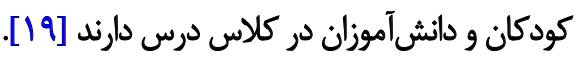

4. Hospodsky
بيوآئروسلها را كزارش كردهاند. براى مثال در مطالعه انجامشده

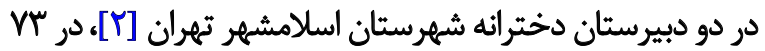

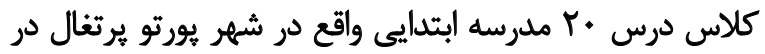

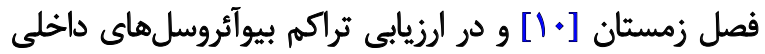

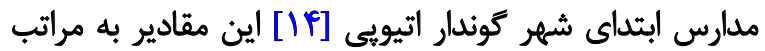

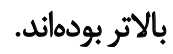

فاكتورهاى مختلف مانئد فصل نمونهبردارى، شرايط محيطى

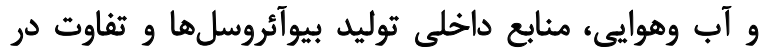

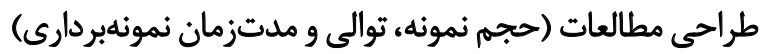

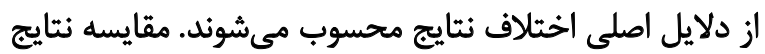

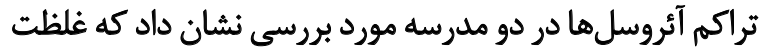

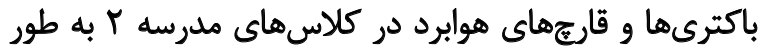

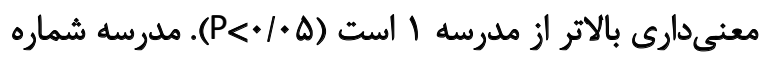

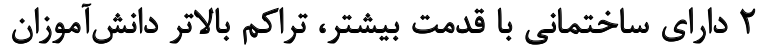

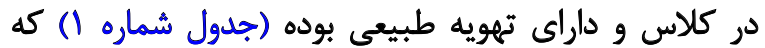
مى توانئد دلايل خوبى براي الفزايش ترائ تراكم بيوآئروسلها

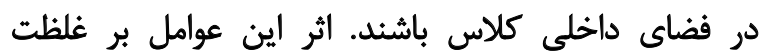

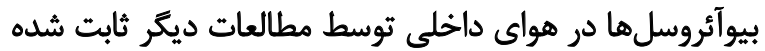

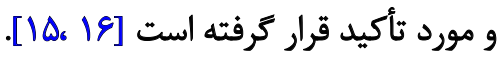

اين تحقيق نشان داد كه غلظت باكترى هاد در مقايسه با قارجها

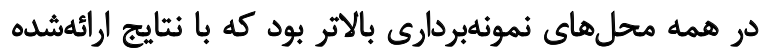

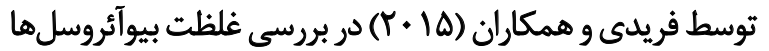

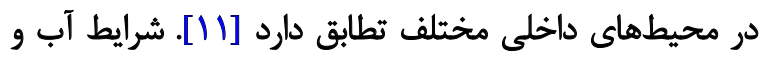

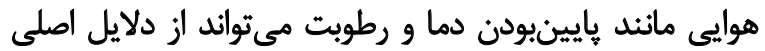

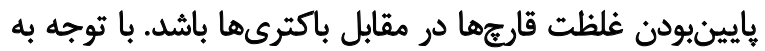

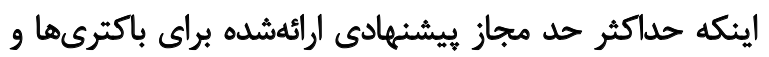

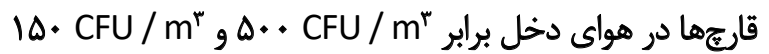

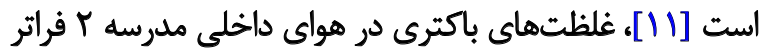

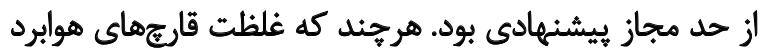

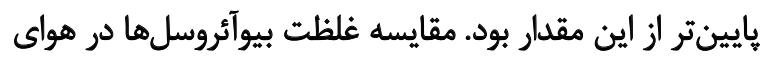

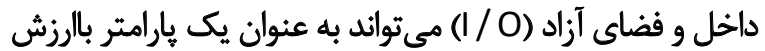

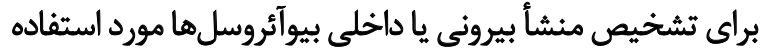

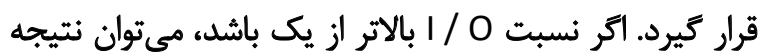

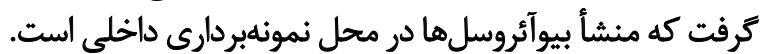

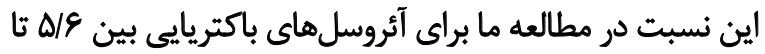

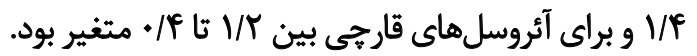

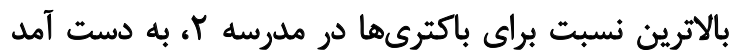

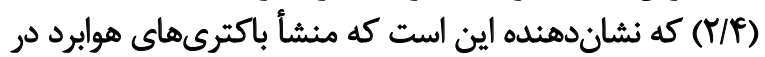

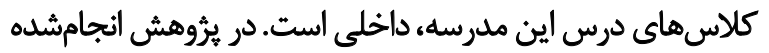

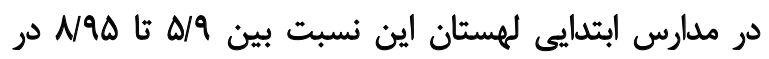

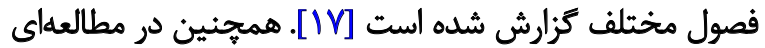

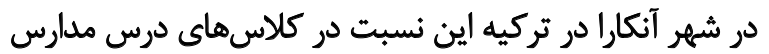

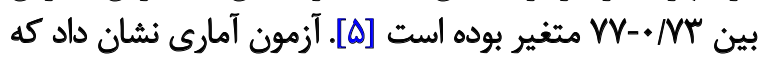




\section{تثيجليرى}

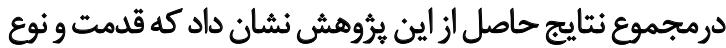

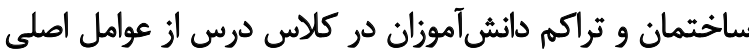

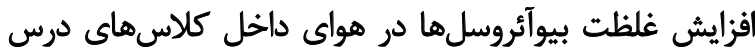

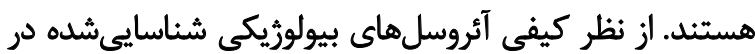

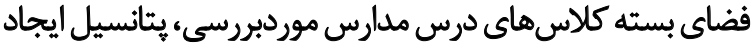

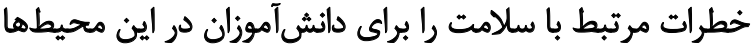

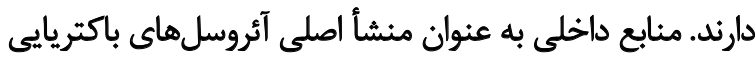

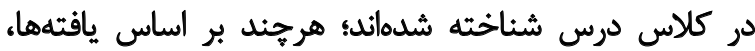

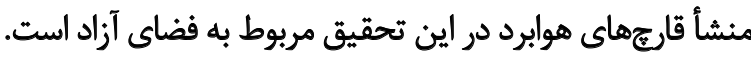

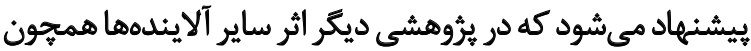
تركيبات آلى فرّار مورد ارزيابى قرار ئيردي

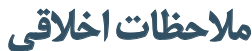

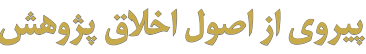

اين مطالعه با كد اخلاق IR.ARAKMU.REC.1397.76 به به يه تصويب كميته اخلاق يُروهشى دانشكاه علوميزئشكى اراك رسيده

$$
\text { مأم }
$$

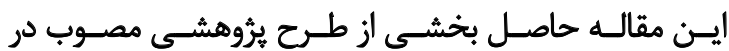

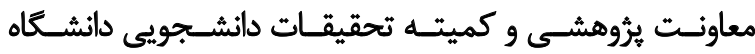

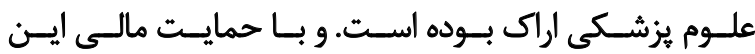

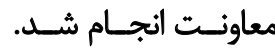

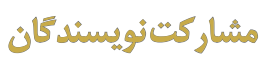

تمامى نويسندكان در نكارش اين مقاله به يك اندازه مشاركت كائشتئ.

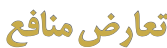

نويسندكان تصريح مى كنيند كه هيجتونه تضاد منافعى براى

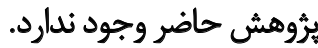

$$
\text { 药 }
$$

اين مقاله حاصل بخشى از طرح برؤشىى مصوب با شماره

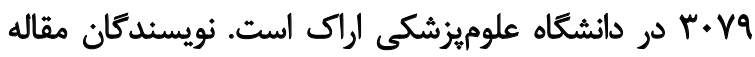

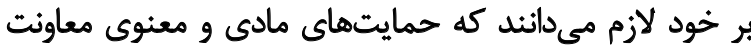

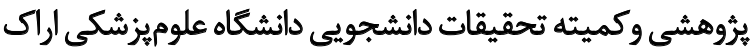

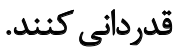




\section{References}

[1] Morawska L, Ayoko GA, Bae GN, Buonanno G, Chao CYH, Clifford S, et al. Airborne particles in indoor environment of homes, schools, offices and aged care facilities: The main routes of exposure. Environ Int. 2017; 108:75-83. [DOI:10.1016/j.envint.2017.07.025] [PMID]

[2] Kashi G. [Investigation of the bio-aerosols concentration from high schools indoor air in Islamshahr county in 1392-3 (Persian)]. . Saf Promot Inj Prev. 2015; 3(1):57-66.

[3] Majd E, McCormack M, Davis M, Curriero F, Berman J, Connolly F, et al. Indoor air quality in inner-city schools and its associations with building characteristics and environmental factors. Environ Res. 2019; 170:83-91. [DOI:10.1016/j.envres.2018.12.012] [PMID]

[4] Brągoszewska E, Mainka A, Pastuszka JS, Lizończyk K, Desta YG. Assessment of bacterial aerosol in a preschool, primary school and high school in Poland. Atmosphere. 2018; 9:87. [DOI:10.3390/atmos9030087]

[5] Mentese S, Rad AY, Arısoy M, Güllü G. Seasonal and spatial variations of bioaerosols in indoor urban environments, Ankara, Turkey. Indoor Built Environ. 2012; 21:797-810. [DOI:10.1177/1420326X11425965]

[6] Mirhoseini SH, Nikaeen M, Satoh K, Makimura K. Assessment of airborne particles in indoor environments: Applicability of particle counting for prediction of bioaerosol concentrations. Aerosol Air Qual Res. 2016; 16:1903-10. [DOI:10.4209/aaqr.2015.08.0528]

[7] Mirhoseini SH, Nikaeen M, Hatamzadeh M, Hassanzadeh A. Assessment of bioaerosol concentration in the indoor environments. Health Syst Res Summer. 2014; 10(2):376-85.

[8] Mirhoseini SH, Nikaeen M, Shamsizadeh Z, Khanahmad H. Hospital air: A potential route for transmission of infections caused by $\beta$-lactamresistant bacteria. Am J Infect Control. 2016; 44:898-904. [DOI:10.1016/j. ajic.2016.01.041] [PMID]

[9] Ehrampoosh MH, ZareSakhvidi MJ, Mehrparvar AH, Soltanianzadeh Z, Gamshidi S, Taherzade S. Evaluating suspended particles concentration of the inside and outside air of the classroom and its influencing factors in middle schools and high schools of yazd. Tolooebehdasht. 2015; 14:11-22.

[10] Madureira J, Paciência I, Pereira C, Teixeira JP, Fernandes E de O. Indoor air quality in Portuguese schools: Levels and sources of pollutants. Indoor Air. 2016; 26:526-37. [DOI:10.1111/ina.12237] [PMID]

[11] Faridi S, Hassanvand MS, Naddafi K, Yunesian M, Nabizadeh R, Sowlat $\mathrm{MH}$, et al. Indoor/outdoor relationships of bioaerosol concentrations in a retirement home and a school dormitory. Environ Sci Pollut Res. 2015; 22:8190-8200. [DOI:10.1007/s11356-014-3944-y] [PMID]

[12] Alves C, Duarte M, Ferreira M, Alves A, Almeida A, Cunha Â. Air quality in a school with dampness and mould problems. Air Qual Atmosphere Health. 2016; 9:107-115. [DOI:10.1007/s11869-015-0319-6]

[13] Mentese S, Tasdibi D. Airborne bacteria levels in indoor urban environments: The influence of season and prevalence of Sick Building Syndrome (SBS). Indoor Built Environ. 2016; 25:563-580. [DOI:10.1177/1420326X14562454]

[14] Andualem Z, Gizaw Z, Bogale L, Dagne H. Indoor bacterial load and its correlation to physical indoor air quality parameters in public primary schools. Multidiscip Respir Med. 2019; 14:2. [DOI:10.1186/s40248-0180167-y] [PMID] [PMCID]

[15] Madureira J, Paciência I, Rufo JC, Pereira C, Teixeira JP, de Oliveira Fernandes $E$. Assessment and determinants of airborne bacterial and fungal concentrations in different indoor environments: Homes, child day- care centres, primary schools and elderly care centres. Atmos Environ. 2015; 109:139-46. [DOI:10.1016/j.atmosenv.2015.03.026]

[16] Mentese S, Arisoy M, Rad AY, Güllü G. Bacteria and fungi levels in various indoor and outdoor environments in Ankara, Turkey. Clean-Soil Air Water. 2009; 37:487-93. [DOI:10.1002/clen.200800220]

[17] Canha N, Almeida SM, do Carmo Freitas M, Wolterbeek HT. Assessment of bioaerosols in urban and rural primary schools using passive and active sampling methodologies. Arch Environ Prot. 2015; 41:11-22. [DOI:10.1515/aep-2015-0034]

[18] Cavaleiro Rufo J, Madureira J, Paciência I, Aguiar L, Pereira C, Silva D, et al. Indoor fungal diversity in primary schools may differently influence allergic sensitization and asthma in children. Pediatr Allergy Immunol. 2017; 28:332-9. [DOI:10.1111/pai.12704] [PMID]

[19] Salonen H, Duchaine C, Mazaheri M, Clifford S, Morawska L. Airborne culturable fungi in naturally ventilated primary school environments in a subtropical climate. Atmos Environ. 2015; 106:412-8. [DOI:10.1016/j. atmosenv.2014.07.052

[20] Mohammadyan M, Alizadeh-Larimi A, Etemadinejad S, Latif MT, Heibati B, Yetilmezsoy K, et al. Particulate air pollution at schools: Indooroutdoor relationship and determinants of indoor concentrations. Aerosol Air Qual Res. 2017; 17:857-64. [DOI:10.4209/aaqr.2016.03.0128]

[21] Hospodsky D, Yamamoto N, Nazaroff WW, Miller D, Gorthala S, Peccia J. Characterizing airborne fungal and bacterial concentrations and emission rates in six occupied children's classrooms. Indoor Air. 2015; 25:641 52. [DOI:10.1111/ina.12172] [PMID]

[22] Deng W, Chai Y, Lin H, So WW, Ho KWK, Tsui AKY, et al. Distribution of bacteria in inhalable particles and its implications for health risks in kindergarten children in Hong Kong. Atmos Environ. 2016; 128:268-75. [DOI:10.1016/j.atmosenv.2016.01.017]

[23] Aydogdu H, Asan A, Otkun MT, Ture M. Monitoring of fungi and bacteria in the indoor air of primary schools in Edirne city, Turkey. Indoor Built Environ. 2005; 14:411-25. [DOI:10.1177/1420326X05057539]

[24] Mirhoseini SH, Nikaeen M, Shamsizadeh Z, Aali R. [Prevalence and molecular identification of antibiotic resistant airborne bacteria at intensive care units (Persian)]. Koomesh. 2018; 20:772-8. 